\title{
AN OPERATIONAL CALCULUS MODEL FOR THE CENTRAL DIFFERENCE AND EXPONENTIAL-TRIGONOMETRIC AND HYPERBOLICFIBONACCI SEQUENCES
}

\section{Hubert Wysocki}

Polish Naval Academy, Faculty of Mechanical and Electrical Engineering, Śmidowicza 69 Str., 81-127 Gdynia, Poland; e-mail: h.wysocki@amw.gdynia.pl

\begin{abstract}
In the paper, there has been constructed such a non-classical Bittner operational calculus model, in which the derivative is understood as a central difference $D_{n}\{x(k)\}:=\{x(k+n)-x(k-n)\}$. The discussed model has been generalized by considering the operation $D_{n, b}\{x(k)\}:=\{x(k+n)-b x(k-n)\}$, where $b \in \mathbb{C} \backslash\{0\}$. In the $D_{1}$-difference model exponential-trigonometric and hyperbolic Fibonacci sequences have been introduced.
\end{abstract}

Key words:

operational calculus, derivative, integrals, limit conditions, central difference, Fibonacci sequences.

Research article

(c) 2018 Hubert Wysocki

This is an open access article licensed under the Creative Commons Attribution-NonCommercial-NoDerivatives 4.0 license (http://creativecommons.org/licenses/by-nc-nd/4.0/) 


\section{INTRODUCTION}

From the integral calculus fundamental theorems [1] it follows that for all functions $\{f(t)\} \in C^{0}([a, b], \mathbb{R}),\{x(t)\} \in C^{1}([a, b], \mathbb{R})$ and for each $t, t_{0} \in[a, b]$ holds the below:

$$
\frac{d}{d t} \int_{t_{0}}^{t} f(\tau) d \tau=f(t), \quad \int_{t_{0}}^{t} x^{\prime}(\tau) d \tau=x(t)-x\left(t_{0}\right)
$$

Hence, if

$$
S\{x(t)\}:=\left\{x^{\prime}(t)\right\}, \quad T_{t_{0}}\{f(t)\}:=\left\{\int_{t_{0}}^{t} f(\tau) d \tau\right\}, \quad s_{t_{0}}\{x(t)\}:=\left.\{x(t)\}\right|_{t=t_{0}} \equiv\left\{x\left(t_{0}\right)\right\},
$$

then on the basis of (1) we get

$$
S T_{t_{0}} f=f, \quad T_{t_{0}} S x=x-s_{t_{0}} x,
$$

where $f=\{f(t)\}, x=\{x(t)\}^{1}$.

In view of the properties (3), the operations (2) are a classic example of the so-called Bittner operational calculus [2-5]. Namely, we say that the system

$$
\left(C^{0}([a, b], \mathbb{R}), C^{1}([a, b], \mathbb{R}), \frac{d}{d t}, \int_{t_{0}}^{t},\left.\right|_{t=t_{0}}, t_{0} \in[a, b]\right)
$$

is a continuous model (representation) of this calculus with the ordinary derivative $S:=d / d t$.

In the difference calculus $[7,10,12]$, to the derivative

$$
x^{\prime}(t) \equiv \frac{d x(t)}{d t}:=\lim _{h \rightarrow 0} \frac{d_{h} x(t)}{d_{h} t},
$$

where

$$
d_{h} x(t):=x(t+h)-x(t)
$$

$1\{f(t)\}$ denotes the symbol of a function $f$, i.e. $f=\{f(t)\}$, whereas $f(t)$ means the value of the function $\{f(t)\}$ for an argument $t$. This denotation is derived from J. Mikusiński [15]. 
there corresponds a forward difference

$$
\Delta x(k):=x(k+1)-x(k) .
$$

In [6] there have been considered operations $S, T_{k_{0}}, s_{k_{0}}$ where $k_{0} \in \mathbb{N}_{0}:=$ $\mathbb{N} \cup\{0\}^{2}$, which are determined on a linear space $C\left(\mathbb{N}_{0}, \mathbb{R}\right)$ of real sequences $x=\{x(k)\}_{k \in \mathbb{N}_{0}}$ with the following formulas

$$
S x \equiv \Delta x, \quad T_{k_{0}} x:=\left\{\sum_{i=0}^{k-1} x(i)-\sum_{i=0}^{k_{0}-1} x(i)\right\}, \quad s_{k_{0}} x:=\left\{x\left(k_{0}\right)\right\} .
$$

They form a discrete model of the Bittner operational calculus, because they possess properties analogical to (3), that is

$$
S T_{k_{0}} x=x, \quad T_{k_{0}} S x=x-s_{k_{0}} x .
$$

A generalization of the above representation is a model with $S$ as an $n^{\text {th }}$ $\operatorname{order}(n \in \mathbb{N})$ forward difference

$$
S x \equiv \Delta_{n} x:=\{x(k+n)-x(k)\},
$$

which was introduced in [22].

A generalization of the ordinary derivative $x^{\prime}(t)$ is the Schwarz derivative $x_{s}^{\prime}(t)$, also known as a symmetric derivative [14]. It is defined by the formula

$$
x_{s}^{\prime}(t):=\lim _{h \rightarrow 0} \frac{\delta_{h} x(t)}{\delta_{h} t}
$$

where

$$
\delta_{h} x(t):=x(t+h)-x(t-h) .
$$

If there exists $x^{\prime}(t)$, then there also exists $x_{s}^{\prime}(t)$ and $x^{\prime}(t)=x_{s}^{\prime}(t)$. Moreover, e.g. for $x(t):=|t|$ we have $x_{s}^{\prime}(0)=0$, while $x^{\prime}(0)$ does not exist [20].

In numerical methods, the symmetric difference $\delta_{h}$ is applied to the approximation of the ordinary derivative $x^{\prime}(t)[13]$. Namely, if $\{x(t)\} \in C^{3}([a, b], \mathbb{R})$, then

$$
x^{\prime}(t) \approx \frac{\delta_{h} x(t)}{2 h} .
$$

$2 \mathbb{N}$ denotes a set of natural numbers.

3 (214) 2018 
Likewise, for $\{x(t)\} \in C^{5}([a, b], \mathbb{R})$ we have

$$
x^{\prime}(t) \approx \frac{8 \delta_{h} x(t)-\delta_{2 h} x(t)}{12 h} .
$$

In the calculus of differences, similarly as before, to the symmetric difference $\delta_{h}$ there corresponds a central difference

$$
D x(k) \equiv D_{1} x(k):=x(k+1)-x(k-1) .
$$

In this paper, we will construct such a discrete model of the Bittner operational calculus, in which the derivative $S$ will be understood as an operation

$$
D_{n}\{x(k)\}:=\{x(k+n)-x(k-n)\}, \quad n \in \mathbb{N}
$$

determined on the space of two-sided sequences, i.e. if $k \in \mathbb{Z}^{3}$. We will determine the operations $T_{k_{0}}$ and $s_{k_{0}}$, where $k_{0} \in \mathbb{Z}$ in such a way that the relations (3) are fulfilled.

\section{FOUNDATIONS OF THE NON-CLASSICAL BITTNER OPERATIONAL CALCULUS}

The Bittner operational calculus is a system

$$
C O\left(L^{0}, L^{1}, S, T_{q}, s_{q}, Q\right)^{4}
$$

where $L^{0}$ and $L^{1}$ are linear spaces (over the same scalar field $\Gamma$ ) such that $L^{1} \subset L^{0}$. A linear operation $S: L^{1} \longrightarrow L^{0}$ (denoted as $S \in \mathscr{L}\left(L^{1}, L^{0}\right)$ ), called a derivative, is a surjection. Moreover, $Q$ is a set of indices $q$ for the operations $T_{q} \in \mathscr{L}\left(L^{0}, L^{1}\right)$ and $s_{q} \in \mathscr{L}\left(L^{1}, L^{1}\right)$ such that $S T_{q} f=f, f \in L^{0}$ and $s_{q} x=x-T_{q} S x, x \in L^{1} . T_{q}$ and $s_{q}$ are called integrals and limit conditions, respectively. The kernel of $S$, i.e. $\operatorname{Ker} S$ is called a set of constants for the derivative $S$. The limit conditions $s_{q}$ are projections of $L^{1}$ onto the subspace $\operatorname{Ker} S$.

By means of induction, we determine a sequence of spaces $L^{n}, n \in \mathbb{N}$ in such a way that

$$
L^{n}:=\left\{x \in L^{n-1}: S x \in L^{n-1}\right\}
$$

$3 \mathbb{Z}$ denotes a set of integer numbers.

${ }^{4}$ The abbreviation $C O$ is derived from the French calcul opératoire (operational calculus). 
Then

$$
\ldots \subset L^{n} \subset L^{n-1} \subset \ldots \subset L^{1} \subset L^{0}
$$

and

$$
S^{n}\left(L^{m+n}\right)=L^{m}
$$

where

$$
\mathscr{L}\left(L^{n}, L^{0}\right) \ni S^{n}:=\underbrace{S \circ S \circ \ldots \circ S}_{n \text {-times }}, \quad m \in \mathbb{N}_{0}, n \in \mathbb{N} .
$$

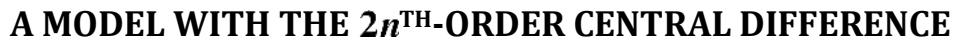

Let $\mathbb{C}$ be a set of complex numbers. What is more, let $C(\mathbb{Z}, \mathbb{C})$ be a linear space of two-sided complex sequences $x=\{x(k)\}_{k \in \mathbb{Z}}$ with usual sequences addition and sequences multiplication by complexes.

The operation (4), determined on $C(\mathbb{Z}, \mathbb{C})$, will be called the $2 n^{\text {th }}$-order central difference, where $n$ is a given natural number.

Let us notice that any element $c \in \operatorname{Ker} D_{n}$ is a $2 n$-periodic sequence, since for each $k \in \mathbb{Z}$ we have

$$
c(k+n)-c(k-n)=0 \Longleftrightarrow c(k+2 n)=c(k) .
$$

Then, for any sequence $c \in \operatorname{Ker} D_{n}$ there exist numbers $a_{0}, a_{1}, \ldots, a_{2 n-1} \in \mathbb{C}$ such that

$$
c=\left\{a_{0} \varepsilon_{0}^{k}+a_{1} \varepsilon_{1}^{k}+\cdots+a_{2 n-1} \varepsilon_{2 n-1}^{k}\right\},
$$

where

$$
\varepsilon_{0}, \varepsilon_{1}, \ldots, \varepsilon_{2 n-1}
$$

are $2 n^{\text {th }}$ roots of unity, i.e.

$$
\varepsilon_{j}=\cos \frac{j \pi}{n}+\mathrm{i} \sin \frac{j \pi}{n}, \quad j \in \overline{0,2 n-1^{5}},
$$

while ' $i$ ' denotes the imaginary unit.

$$
5 \overline{0,2 n-1}:=\{0,1, \ldots, 2 n-1\} .
$$

3 (214) 2018 
In what follows, we will use the below properties of the sequence (6):

$$
\begin{gathered}
\varepsilon_{j}^{k+2 \ell n}=\varepsilon_{j}^{k}, \quad j \in \overline{0,2 n-1}, k, \ell \in \mathbb{Z}, \\
\varepsilon_{0}^{m}+\varepsilon_{1}^{m}+\ldots+\varepsilon_{2 n-1}^{m}=0, \quad m \neq 2 \ell n, \ell, m \in \mathbb{Z}, n \in \mathbb{N} .
\end{gathered}
$$

We will prove the following

Theorem. The system (5), where $x=\{x(k)\} \in L^{0}=L^{1}:=C(\mathbb{Z}, \mathbb{C}), k_{0} \equiv q \in$ $Q:=\mathbb{Z}$ and

$$
T_{k_{0}} x:=\left\{\begin{array}{c}
S x:=\{x(k+n)-x(k-n)\}, \\
-\frac{1}{2 n} \sum_{j=0}^{2 n-1} \sum_{i=k+n}^{k_{0}+n-1} \varepsilon_{j}^{k+n-i} x(i) \text { for } k<k_{0} \\
\frac{1}{2 n} \sum_{j=0}^{2 n-1} \sum_{i=k_{0}+n}^{k+n-1} \varepsilon_{j}^{k+n-i} x(i) \text { for } k=k_{0} \quad, \quad k \in \mathbb{Z}, \\
s_{k_{0}} x:=\left\{\frac{1}{2 n} \sum_{j=0}^{2 n-1} \sum_{i=k_{0}}^{k_{0}+2 n-1} \varepsilon_{j}^{k-i} x(i)\right\}
\end{array}\right.
$$

forms a discrete model of the Bittner operational calculus ${ }^{6}$.

Proof. It is obvious that the operations (7)-(9) are linear. Let $\{y(k)\}:=T_{k_{0}}\{x(k)\}$. Then, for $k=k_{0}$ we obtain

$$
\begin{gathered}
\left.S\{y(k)\}\right|_{k=k_{0}}=\left\{y\left(k_{0}+n\right)-y\left(k_{0}-n\right)\right\} \\
=\left\{\frac{1}{2 n} \sum_{j=0}^{2 n-1} \sum_{i=k_{0}+n}^{k_{0}+2 n-1} \varepsilon_{j}^{k_{0}-i} x(i)+\frac{1}{2 n} \sum_{j=0}^{2 n-1} \sum_{i=k_{0}}^{k_{0}+n-1} \varepsilon_{j}^{k_{0}-i} x(i)\right\} \\
=\left\{\frac{1}{2 n} \sum_{j=0}^{2 n-1} \sum_{i=k_{0}}^{k_{0}+2 n-1} \varepsilon_{j}^{k_{0}-i} x(i)\right\} \\
=\left\{x\left(k_{0}\right)+\frac{1}{2 n} \sum_{i=k_{0}+1}^{k_{0}+2 n-1}\left(\varepsilon_{0}^{k_{0}-i}+\varepsilon_{1}^{k_{0}-i}+\cdots+\varepsilon_{2 n-1}^{k_{0}-i}\right) x(i)\right\}=\left.\{x(k)\}\right|_{k=k_{0}} .
\end{gathered}
$$

${ }^{6}$ Given the definition of integrals $T_{k_{0}}$, we assume that $\sum_{i=k_{0}+n}^{k_{0}+n-1} f(i):=0$. 
For $k<k_{0}$ and $k+n=k_{0}$, i.e. $k=k_{0}-n$, we get in turn

$$
\begin{gathered}
S\{y(k)\}=\{y(k+n)-y(k-n)\}=\left\{y\left(k_{0}\right)-y\left(k_{0}-2 n\right)\right\} \\
=\left\{0+\frac{1}{2 n} \sum_{j=0}^{2 n-1} \sum_{i=k_{0}-n}^{k_{0}+n-1} \varepsilon_{j}^{k_{0}-n-i} x(i)\right\} \\
=\left\{x\left(k_{0}-n\right)+\frac{1}{2 n} \sum_{i=k_{0}-n+1}^{k_{0}+n-1}\left(\varepsilon_{0}^{k_{0}-n-i}+\varepsilon_{1}^{k_{0}-n-i}+\cdots+\varepsilon_{2 n-1}^{k_{0}-n-i}\right) x(i)\right\}=\left\{x\left(k_{0}-n\right)\right\} .
\end{gathered}
$$

Therefore,

$$
S\{y(k)\}=\left\{x\left(k_{0}-n\right)\right\}, \text { that is } S\{y(k)\}=\{x(k)\} .
$$

For $k<k_{0}$ and $k+n<k_{0}$, i.e. $k<k_{0}-n$, we have

$$
\begin{gathered}
S\{y(k)\}=\{y(k+n)-y(k-n)\} \\
=\left\{\frac{1}{2 n} \sum_{j=0}^{2 n-1} \sum_{i=k}^{k_{0}+n-1} \varepsilon_{j}^{k-i} x(i)-\frac{1}{2 n} \sum_{j=0}^{2 n-1} \sum_{i=k+2 n}^{k_{0}+n-1} \varepsilon_{j}^{k-i} x(i)\right\} \\
=\left\{\frac{1}{2 n} \sum_{j=0}^{2 n-1} \sum_{i=k}^{k+2 n-1} \varepsilon_{j}^{k-i} x(i)\right\} .
\end{gathered}
$$

Hence, similarly as in (10), we conclude that $S\{y(k)\}=\{x(k)\}$.

If $k<k_{0}$ and $k+n>k_{0}$, i.e. $k_{0}-n<k<k_{0}$, we get in turn

$$
\begin{gathered}
S\{y(k)\}=\{y(k+n)-y(k-n)\} \\
=\left\{\frac{1}{2 n} \sum_{j=0}^{2 n-1} \sum_{i=k_{0}+n}^{k+2 n-1} \varepsilon_{j}^{k-i} x(i)+\frac{1}{2 n} \sum_{j=0}^{2 n-1} \sum_{i=k}^{k_{0}+n-1} \varepsilon_{j}^{k-i} x(i)\right\} \\
=\left\{\frac{1}{2 n} \sum_{j=0}^{2 n-1} \sum_{i=k}^{k+2 n-1} \varepsilon_{j}^{k-i} x(i)\right\} .
\end{gathered}
$$

So, by analogy to (11), we have $S\{y(k)\}=\{x(k)\}$.

If $k>k_{0}$ and $k-n=k_{0}$, i.e. $k=k_{0}+n$, then 


$$
\begin{gathered}
S\{y(k)\}=\{y(k+n)-y(k-n)\}=\left\{y\left(k_{0}+2 n\right)-y\left(k_{0}\right)\right\} \\
=\left\{\frac{1}{2 n} \sum_{j=0}^{2 n-1} \sum_{i=k_{0}+n}^{k_{0}+3 n-1} \varepsilon_{j}^{k_{0}+n-i} x(i)-0\right\} \\
=\left\{x\left(k_{0}+n\right)+\frac{1}{2 n} \sum_{i=k_{0}+n+1}^{k_{0}+3 n-1}\left(\varepsilon_{0}^{k_{0}+n-i}+\varepsilon_{1}^{k_{0}+n-i}+\cdots+\varepsilon_{2 n-1}^{k_{0}+n-i}\right) x(i)\right\}=\left\{x\left(k_{0}+n\right)\right\},
\end{gathered}
$$

that is $S\{y(k)\}=\{x(k)\}$.

For $k>k_{0}$ and $k-n>k_{0}$, i.e. $k>k_{0}+n$, we obtain

$$
\begin{gathered}
S\{y(k)\}=\{y(k+n)-y(k-n)\} \\
=\left\{\frac{1}{2 n} \sum_{j=0}^{2 n-1} \sum_{i=k_{0}+n}^{k+2 n-1} \varepsilon_{j}^{k-i} x(i)-\frac{1}{2 n} \sum_{j=0}^{2 n-1} \sum_{i=k_{0}+n}^{k-1} \varepsilon_{j}^{k-i} x(i)\right\} \\
=\left\{\frac{1}{2 n} \sum_{j=0}^{2 n-1} \sum_{i=k}^{k+2 n-1} \varepsilon_{j}^{k-i} x(i)\right\},
\end{gathered}
$$

which, similarly as in (11), also means that $S\{y(k)\}=\{x(k)\}$.

Lastly, if $k>k_{0}$ and $k-n<k_{0}$, i.e. $k_{0}<k<k_{0}+n$, then

$$
\begin{gathered}
S\{y(k)\}=\{y(k+n)-y(k-n)\} \\
=\left\{\frac{1}{2 n} \sum_{j=0}^{2 n-1} \sum_{i=k_{0}+n}^{k+2 n-1} \varepsilon_{j}^{k-i} x(i)+\frac{1}{2 n} \sum_{j=0}^{2 n-1} \sum_{i=k}^{k_{0}+n-1} \varepsilon_{j}^{k-i} x(i)\right\} \\
=\left\{\frac{1}{2 n} \sum_{j=0}^{2 n-1} \sum_{i=k}^{k+2 n-1} \varepsilon_{j}^{k-i} x(i)\right\}=\{x(k)\} .
\end{gathered}
$$

Finally, we can conclude that the property $S T_{k_{0}} x=x$ is fulfilled.

Let $\{f(k)\}:=S\{x(k)\}=\{x(k+n)-x(k-n)\}$. Therefore, for $k<k_{0}$ we get

$$
\begin{aligned}
& T_{k_{0}} S\{x(k)\}=T_{k_{0}}\{f(k)\}=\left\{-\frac{1}{2 n} \sum_{j=0}^{2 n-1} \sum_{i=k+n}^{k_{0}+n-1} \varepsilon_{j}^{k+n-i} f(i)\right\} \\
= & \left\{\frac{1}{2 n} \sum_{j=0}^{2 n-1}\left[\sum_{i=k+n}^{k_{0}+n-1} \varepsilon_{j}^{k+n-i} x(i-n)-\sum_{i=k+n}^{k_{0}+n-1} \varepsilon_{j}^{k+n-i} x(i+n)\right]\right\}
\end{aligned}
$$




$$
\begin{gathered}
=\left\{\frac { 1 } { 2 n } \sum _ { j = 0 } ^ { 2 n - 1 } \left[\sum_{i=k}^{k_{0}-1} \varepsilon_{j}^{k-i} x(i)+\sum_{i=k_{0}}^{k+2 n-1} \varepsilon_{j}^{k-i} x(i)\right.\right. \\
\left.\left.-\left(\sum_{i=k_{0}}^{k+2 n-1} \varepsilon_{j}^{k-i} x(i)+\sum_{i=k+2 n}^{k_{0}+2 n-1} \varepsilon_{j}^{k-i} x(i)\right)\right]\right\} \\
=\left\{\frac{1}{2 n} \sum_{j=0}^{2 n-1} \sum_{i=k}^{k+2 n-1} \varepsilon_{j}^{k-i} x(i)\right\}-\left\{\frac{1}{2 n} \sum_{j=0}^{2 n-1} \sum_{i=k_{0}}^{k_{0}+2 n-1} \varepsilon_{j}^{k-i} x(i)\right\} .
\end{gathered}
$$

Hence, on the basis of (11), we eventually obtain

$$
T_{k_{0}} S\{x(k)\}=\{x(k)\}-\left\{\frac{1}{2 n} \sum_{j=0}^{2 n-1} \sum_{i=k_{0}}^{k_{0}+2 n-1} \varepsilon_{j}^{k-i} x(i)\right\}=\{x(k)\}-s_{k_{0}}\{x(k)\} .
$$

Likewise, if $k>k_{0}$, then

$$
\begin{gathered}
T_{k_{0}} S\{x(k)\}=T_{k_{0}}\{f(k)\}=\left\{\frac{1}{2 n} \sum_{j=0}^{2 n-1} \sum_{i=k_{0}+n}^{k+n-1} \varepsilon_{j}^{k+n-i} f(i)\right\} \\
=\left\{\frac{1}{2 n} \sum_{j=0}^{2 n-1}\left[\sum_{i=k_{0}+n}^{k+n-1} \varepsilon_{j}^{k+n-i} x(i+n)-\sum_{i=k_{0}+n}^{k+n-1} \varepsilon_{j}^{k+n-i} x(i-n)\right]\right\} \\
=\left\{\frac { 1 } { 2 n } \sum _ { j = 0 } ^ { 2 n - 1 } \left[\sum_{i=k}^{k_{0}+2 n-1} \varepsilon_{j}^{k-i} x(i)+\sum_{i=k_{0}+2 n}^{k+2 n-1} \varepsilon_{j}^{k-i} x(i)\right.\right. \\
\left.\left.=\left\{\frac{1}{2 n} \sum_{j=0}^{2 n-1} \sum_{i=k}^{k+2 n-1} \varepsilon_{j}^{k-i} x(i)\right\}-\left\{\frac{1}{2 n} \sum_{j=0}^{k-1} \sum_{i=k_{0}}^{2 n-1} \varepsilon_{j}^{k-i} x(i)+\sum_{i=k}^{k_{0}+2 n-1} \varepsilon_{j}^{k-i} x(i)\right)\right]\right\}
\end{gathered}
$$

Therefore, the property $T_{k_{0}} S x=x-s_{k_{0}} x$ is also fulfilled.

Since for $k \in \overline{k_{0}+1, k_{0}+2 n-1}$ we have

$$
\sum_{i=k_{0}+n}^{k+n-1}\left(\varepsilon_{0}^{k+n-i}+\varepsilon_{1}^{k+n-i}+\cdots+\varepsilon_{2 n-1}^{k+n-i}\right)=0,
$$

then, from the definition (8) of integrals $T_{k_{0}}$, we get the following 
Corollary 1. If $\{y(k)\}:=T_{k_{0}}\{x(k)\}$, then

$$
y(k)=0 \text { for } k \in \overline{k_{0}, k_{0}+2 n-1} .
$$

Corollary 2. The numbers $x\left(k_{0}\right), x\left(k_{0}+1\right), \ldots, x\left(k_{0}+2 n-1\right)$ form a cycle of the 2 -periodic sequence $\{c(k)\}=s_{k_{0}}\{x(k)\}$, i.e.

$$
c(k)=c(k+2 \ell n)=x(k), \quad k \in \overline{k_{0}, k_{0}+2 n-1}, \ell \in \mathbb{Z} .
$$

Moreover, $s_{k_{0}} T_{k_{0}}\{x(k)\}=\{0\}$ and $s_{k_{0}}\{c(k)\}=\{c(k)\}$.

Example. Using the Mathematica ${ }^{\circledR}$ program, we will list the terms of the sequence $\{y(k)\}:=T_{k_{0}}\{x(k)\}$ for $k \in \overline{-22,19}$ in the case when $n=2$ and $k_{0}=-2$. By means of (8) we obtain:

$$
\begin{array}{rl}
k & y(k) \\
\hline-22 & -x(-20)-x(-16)-x(-12)-x(-8)-x(-4) \\
-21 & -x(-19)-x(-15)-x(-11)-x(-7)-x(-3) \\
-20 & -x(-18)-x(-14)-x(-10)-x(-6)-x(-2) \\
-19 & -x(-17)-x(-13)-x(-9)-x(-5)-x(-1) \\
-18 & -x(-16)-x(-12)-x(-8)-x(-4) \\
-17 & -x(-15)-x(-11)-x(-7)-x(-3) \\
-16 & -x(-14)-x(-10)-x(-6)-x(-2) \\
-15 & -x(-13)-x(-9)-x(-5)-x(-1) \\
-14 & -x(-12)-x(-8)-x(-4) \\
-13 & -x(-11)-x(-7)-x(-3) \\
-12 & -x(-10)-x(-6)-x(-2) \\
-11 & -x(-9)-x(-5)-x(-1) \\
-10 & -x(-8)-x(-4) \\
-9 & -x(-7)-x(-3) \\
-8 & -x(-6)-x(-2) \\
-7 & -x(-5)-x(-1) \\
-6 & -x(-4) \\
-5 & -x(-3) \\
-4 & -x(-2) \\
-3 & -x(-1) \\
-2 & 0
\end{array}
$$

\begin{tabular}{rl}
$k$ & $y(k)$ \\
\hline-1 & 0 \\
0 & 0 \\
1 & 0 \\
2 & $x(0)$ \\
3 & $x(1)$ \\
4 & $x(2)$ \\
5 & $x(3)$ \\
6 & $x(0)+x(4)$ \\
7 & $x(1)+x(5)$ \\
8 & $x(2)+x(6)$ \\
9 & $x(3)+x(7)$ \\
10 & $x(0)+x(4)+x(8)$ \\
11 & $x(1)+x(5)+x(9)$ \\
12 & $x(2)+x(6)+x(10)$ \\
13 & $x(3)+x(7)+x(11)$ \\
14 & $x(0)+x(4)+x(8)+x(12)$ \\
15 & $x(1)+x(5)+x(9)+x(13)$ \\
16 & $x(2)+x(6)+x(10)+x(14)$ \\
17 & $x(3)+x(7)+x(11)+x(15)$ \\
18 & $x(0)+x(4)+x(8)+x(12)+x(16)$ \\
19 & $x(1)+x(5)+x(9)+x(13)+x(17)$
\end{tabular}

\section{A CERTAIN GENERALIZATION}

The operation

$$
S_{b}\{x(k)\}:=\{x(k+n)-b x(k-n)\},
$$

where $\{x(k)\} \in L^{0}=L^{1}:=C(\mathbb{Z}, \mathbb{C}), b \in \mathbb{C} \backslash\{0\}$, is a generalization of the central difference (7). 


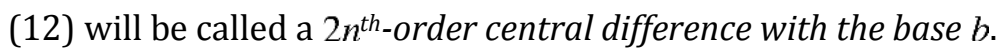

While constructing an operational calculus model corresponding to the derivative (12), we will use the method of solving the equation $x(k+1)-b(k) x(k)=f(k)$ described in [12], as well as the following auxiliary theorems:

Lemma 1 (Th. 3 [5]). An abstract differential equation

$$
S x=f, \quad f \in L^{0}, x \in L^{1}
$$

with the limit condition

$$
s_{q} x=x_{0, q}, \quad x_{0, q} \in \operatorname{Ker} S
$$

has exactly one solution

$$
x=x_{0, q}+T_{q} f .
$$

Lemma 2 (Th. 4 [5]). With a given derivative $S \in \mathscr{L}\left(L^{1}, L^{0}\right)$, the projection $s_{q} \in \mathscr{L}\left(L^{1}, \operatorname{Ker} S\right)$ determines the integral $T_{q} \in \mathscr{L}\left(L^{0}, L^{1}\right)$ from the condition

$$
x=T_{q} f \text { if and only if } S x=f, s_{q} x=0 .
$$

Moreover, the projection $s_{q}$ is a limit condition corresponding to the integral $T_{q}$.

One of the elements of $\operatorname{Ker} S_{b}$ is the sequence

$$
e(k):=b^{\frac{k}{2 n}}, \quad k \in \mathbb{Z} .
$$

Then

$$
e(k+n)=b e(k-n), \quad k \in \mathbb{Z} .
$$

Let us consider the following difference equation

$$
S_{b}\{x(k)\}=\{f(k)\},
$$

i.e.

$$
x(k+n)-b x(k-n)=f(k), \quad k \in \mathbb{Z} .
$$

Hence, we have

$$
\frac{x(k+n)}{e(k+n)}-\frac{x(k-n)}{e(k-n)}=\frac{f(k)}{e(k+n)}, \quad k \in \mathbb{Z},
$$

so

$$
y(k+n)-y(k-n)=g(k), \quad k \in \mathbb{Z},
$$

3 (214) 2018 
where

$$
y(k):=\frac{x(k)}{e(k)}, \quad g(k):=\frac{f(k)}{e(k+n)}, \quad k \in \mathbb{Z} .
$$

The equation (14) can be presented in the form of

$$
S\{y(k)\}=\{g(k)\},
$$

where $S \equiv D_{n}$ is the operation (7).

From Lemma 1 it follows that the solution of the equation (16) is a sequence

$$
\{y(k)\}=s_{k_{0}}\{y(k)\}+T_{k_{0}}\{g(k)\},
$$

where $T_{k_{0}}$ and $s_{k_{0}}$ are operations (8) and (9).

From (15) we get $x(k)=e(k) y(k), k \in \mathbb{Z}$. Finally,

$$
\{x(k)\}=\{e(k)\} s_{k_{0}}\left\{\frac{x(k)}{e(k)}\right\}+\{e(k)\} T_{k_{0}}\left\{\frac{f(k)}{e(k+n)}\right\}
$$

is a solution of (13).

If

$$
\{\widetilde{c}(k)\}:=s_{k_{0}}\left\{\frac{x(k)}{e(k)}\right\}
$$

then $\{\widetilde{c}(k)\} \in \operatorname{Ker} S$, thus

$$
\widetilde{c}(k+n)-\widetilde{c}(k-n)=0, \quad k \in \mathbb{Z} .
$$

Let

$$
s_{b, k_{0}}\{x(k)\}:=\{e(k)\} s_{k_{0}}\left\{\frac{x(k)}{e(k)}\right\}, \quad k_{0} \in Q:=\mathbb{Z},\{x(k)\} \in L^{1} .
$$

Therefore, for each $k \in \mathbb{Z}$ we obtain

$$
\begin{aligned}
& S_{b} s_{b, k_{0}} x(k)=e(k+n) \widetilde{c}(k+n)-b e(k-n) \widetilde{c}(k-n) \\
& =e(k+n)(\widetilde{c}(k+n)-\widetilde{c}(k-n))=e(k+n) \cdot 0=0,
\end{aligned}
$$

that is $s_{b, k_{0}} \in \mathscr{L}\left(L^{1}, \operatorname{Ker} S_{b}\right)$. Moreover, since $s_{k_{0}}\{\widetilde{c}(k)\}=\{\widetilde{c}(k)\}$, then for each $k \in \mathbb{Z}$ we have

$$
\begin{gathered}
s_{b, k_{0}}^{2} x(k)=s_{b, k_{0}}[e(k) \widetilde{c}(k)]=e(k) s_{k_{0}}\left[\frac{e(k) \widetilde{c}(k)}{e(k)}\right] \\
=e(k) s_{k_{0}} \widetilde{c}(k)=e(k) \widetilde{c}(k)=s_{b, k_{0}} x(k) .
\end{gathered}
$$


Finally, $s_{b, k_{0}}$ is a projection of $L^{1}$ onto $\operatorname{Ker} S_{b}$ for each $k_{0} \in \mathbb{Z}$. From Lemma 2 it follows that the projection $s_{b, k_{0}}$ determines an integral $T_{b, k_{0}}$ from the formula (17). Namely,

$$
T_{b, k_{0}}\{f(k)\}:=\{e(k)\} T_{k_{0}}\left\{\frac{f(k)}{e(k+n)}\right\}, \quad k_{0} \in Q,\{f(k)\} \in L^{0} .
$$

What is more, $s_{b, k_{0}}$ is a limit condition corresponding to the integral (19). Hence, we arrive at the

Corollary 3. The system (12), (18), (19) forms a discrete model of the Bittner operational calculus

$$
C O\left(C(\mathbb{Z}, \mathbb{C}), C(\mathbb{Z}, \mathbb{C}), S_{b}, T_{b, k_{0}}, s_{b, k_{0}}, \mathbb{Z}\right) .
$$

In particular, to the derivative $\bar{S}:=\frac{1}{2} S_{-1}$ understood as a $2 n^{\text {th }}$-order central mean

$$
\bar{S}\{x(k)\} \equiv M\{x(k)\}=\left\{\frac{x(k-n)+x(k+n)}{2}\right\},
$$

there correspond the below integrals and limit conditions

$$
\bar{T}_{k_{0}}=2 T_{-1, k_{0}}, \quad \bar{s}_{k_{0}}=s_{-1, k_{0}} .
$$

\section{THE $\lambda$-FIBONACCI SEQUENCES}

A special case of the $2 n^{\text {th }}$-order central difference is a derivative

$$
S x \equiv D_{1} x=\{x(k+1)-x(k-1)\},
$$

to which, in line with Theorem 1 (cf. [21]), there correspond the following integrals

$$
T_{k_{0}} x:=\left\{\begin{aligned}
-\frac{1}{2} \sum_{i=k+1}^{k_{0}}\left[1-(-1)^{k-i}\right] x(i) & \text { for } k<k_{0} \\
0 & \text { for } k=k_{0}, \quad k \in \mathbb{Z} \\
\frac{1}{2} \sum_{i=k_{0}+1}^{k}\left[1-(-1)^{k-i}\right] x(i) & \text { for } k>k_{0}
\end{aligned}\right.
$$

and limit conditions

$$
s_{k_{0}} x:=\left\{\frac{1}{2}\left[x\left(k_{0}\right)+x\left(k_{0}+1\right)\right]+\frac{1}{2}(-1)^{k-k_{0}}\left[x\left(k_{0}\right)-x\left(k_{0}+1\right)\right]\right\} .
$$

3 (214) 2018 
Let $C(\mathbb{Z}, \mathbb{R})$ be a space of two-sided real sequences. For $x, y \in C(\mathbb{Z}, \mathbb{R})$ and $z:=x+y \mathrm{i}$, we define $S z:=S x+\mathrm{i} S y$. Similarly, we determine $T_{k_{0}} z$ and $s_{k_{0}} z$.

A sequence $z=\{z(k)\}$, which is a solution of the problem

$$
\left\{\begin{array}{ll}
S z=\lambda z, & \lambda \in \mathbb{C} \\
s_{k_{0}} z=c, & c \in \operatorname{Ker} S
\end{array},\right.
$$

will be called an exponential element (with $\lambda$ exponent) corresponding to the derivative $(21)^{7}$ (cf. [5]).

This element is determined uniquely, because the only solution of the problem (24) for $c=0$ is $z=0$.

Let us consider such a case of (24), in which the limit condition for $k_{0}=0$ is induced by the initial conditions

$$
z(0)=0, \quad z(1)=1 .
$$

Then, the problem (24) will take the form of

$$
\left\{\begin{array}{l}
z(k+1)-z(k-1)=\lambda z(k) \\
s_{0} z(k)=\frac{1}{2}\left[1-(-1)^{k}\right]
\end{array}, \quad k \in \mathbb{Z}\right.
$$

and for $\lambda \neq \pm 2 \mathrm{i}$ has a solution given by the $\lambda$-Binet formula

$$
z(k)=\frac{\Phi_{\lambda}^{k}-\varphi_{\lambda}^{k}}{\Phi_{\lambda}-\varphi_{\lambda}}, \quad k \in \mathbb{Z},
$$

where

$$
\Phi_{\lambda}:=\frac{\lambda+\sqrt{\lambda^{2}+4}}{2}, \quad \varphi_{\lambda}:=\frac{\lambda-\sqrt{\lambda^{2}+4}}{2} .
$$

The solution (25) is called a $\lambda$-Fibonacci sequence.

Real $\lambda$-Fibonacci sequences for $\lambda \in \mathbb{R}_{>0}$ and $k \in \mathbb{Z}$ were already considered by Gazalé [9] and Stakhov [18], while for $\lambda \in \mathbb{N}$ and $k \in \mathbb{N}_{0}$ - by Falcón and Plaza [8].

The number sequence $\left\{\Phi_{\lambda}\right\}_{\lambda \in \mathbb{N}}$ was called a family of metallic means (proportions) by de Spinadel in $[16,17]$. For $\lambda=1,2,3$ the proportions are called golden, silver and bronze, respectively. tive $S:=d / d t$.

${ }^{7}$ By analogy to the exponential function $\left\{e^{\lambda\left(t-t_{0}\right)} c\right\}$ corresponding to the ordinary deriva- 
Let $\lambda:=\alpha+\beta \mathrm{i}$, where $\alpha^{2}+\beta^{2}>0$. By separating real and imaginary parts in the problem (24), we obtain a system of equations

$$
\left\{\begin{array}{l}
S x=\alpha x-\beta y \\
S y=\beta x+\alpha y
\end{array}\right.
$$

with limit conditions

$$
s_{0} x=c, s_{0} y=0,
$$

where $c=\left\{\frac{1}{2}\left[1-(-1)^{k}\right]\right\}$.

From (26), (27) it follows that the sequences $x, y$ are the solutions of the equation

$$
\left[(S-\alpha I)^{2}+\beta^{2} I\right] u=0^{8}
$$

with the corresponding limit conditions

$$
\begin{aligned}
& s_{0} x=c, s_{0} S x=\alpha c \\
& s_{0} y=0, s_{0} S y=\beta c .
\end{aligned}
$$

Let us denote these solutions as

$$
x:=\{\operatorname{Exp}(\alpha) \operatorname{Cos}(\beta) F(k)\}, \quad y:=\{\operatorname{Exp}(\alpha) \operatorname{Sin}(\beta) F(k)\}
$$

and let us call them exponential-trigonometric Fibonacci sequences.

Likewise, let

$$
z=x+y \mathrm{i}=:\{\operatorname{Exp}(\alpha+\beta \mathrm{i}) F(k)\}
$$

denote an $(\alpha+\beta \mathrm{i})$-Fibonacci sequence. This sequence is an exponential element with the exponent $\lambda=\alpha+\beta$ i and it corresponds to the central difference (21).

Hence, we get the Euler-Fibonacci formula (cf. [5])

$$
\operatorname{Exp}(\alpha+\beta \mathrm{i}) F(k)=\operatorname{Exp}(\alpha) \operatorname{Cos}(\beta) F(k)+\mathrm{i} \operatorname{Exp}(\alpha) \operatorname{Sin}(\beta) F(k), \quad k \in \mathbb{Z},
$$

which is an analogon of the classic Euler formula.

We will use the equivalent notations

${ }^{8} /$ is the identity operation defined on $L^{0}=C(\mathbb{Z}, \mathbb{R})$.

3 (214) 2018 


$$
\begin{gathered}
\operatorname{Exp} F(k) \equiv \operatorname{Exp}(1) F(k), \\
\operatorname{Exp} \operatorname{Sin} F(k) \equiv \operatorname{Exp}(1) \operatorname{Sin}(1) F(k), \\
\operatorname{Exp} \operatorname{Cos} F(k) \equiv \operatorname{Exp}(1) \operatorname{Cos}(1) F(k), \\
\operatorname{Sin} F(k) \equiv \operatorname{Exp}(0) \operatorname{Sin}(1) F(k), \\
\operatorname{Cos} F(k) \equiv \operatorname{Exp}(0) \operatorname{Cos}(1) F(k),
\end{gathered}
$$

whereby the sequences

$$
\{\operatorname{Cos} F(k)\}, \quad\{\operatorname{Sin} F(k)\}
$$

will be called a Fibonacci cosine and sine, respectively.

By means of $\{\operatorname{Exp}(\lambda) F(k)\}$, we can also determine the below hyperbolic Fibonacci sequences (cosine and sine)

$$
\begin{aligned}
& \operatorname{Cosh} F(k):=\frac{\operatorname{Exp}(1) F(k)+\operatorname{Exp}(-1) F(k)}{2} \\
& \operatorname{Sinh} F(k):=\frac{\operatorname{Exp}(1) F(k)-\operatorname{Exp}(-1) F(k)}{2}
\end{aligned}
$$

Then, using the Binet formula (25), we obtain

$$
\begin{aligned}
& \operatorname{Cosh} F(k)= \begin{cases}0 & \text { for } k=2 \ell \\
\operatorname{ch} F \mathrm{~s}(k) & \text { for } k=2 \ell+1\end{cases} \\
& \operatorname{Sinh} F(k)=\left\{\begin{array}{lll}
\operatorname{sh} F \mathrm{~s}(k) & \text { for } k=2 \ell \\
0 & \text { for } & k=2 \ell+1
\end{array}\right.
\end{aligned}
$$

where

$$
\operatorname{ch} F \mathrm{~s}(k):=\frac{\Phi_{1}^{k}+\Phi_{1}^{-k}}{\sqrt{5}}, \quad \operatorname{sh} F \mathrm{~s}(k):=\frac{\Phi_{1}^{k}-\Phi_{1}^{-k}}{\sqrt{5}}, \quad k \in \mathbb{Z}
$$

are so-called symmetric hyperbolic Fibonacci (cosine and sine) sequences, introduced by Stakhov and Rozin in [19] (see also [18]).

Then, we have

$$
F(k)=\operatorname{Sinh} F(k)+\operatorname{Cosh} F(k), \quad k \in \mathbb{Z},
$$

where $\{F(k)\}$ is a classic two-sided 1-Fibonacci sequence, whereby $F(-k)=(-1)^{k+1} F(k), k \in \mathbb{N}$ :

\begin{tabular}{c|rrrrrrrrrrrrrrrrr}
$k$ & $\ldots$ & -7 & -6 & -5 & -4 & -3 & -2 & -1 & 0 & 1 & 2 & 3 & 4 & 5 & 6 & 7 & $\ldots$ \\
\hline$F(k)$ & $\ldots$ & 13 & -8 & 5 & -3 & 2 & -1 & 1 & 0 & 1 & 1 & 2 & 3 & 5 & 8 & 13 & $\ldots$
\end{tabular}


We will now discuss a special case of $\lambda$-Fibonacci sequences, when $\lambda:=1+\mathrm{i}$. From (28)-(30) it follows that $x=\{\operatorname{Exp} \operatorname{Cos} F(k)\}$ and $y=\{\operatorname{Exp} \operatorname{Sin} F(k)\}$ are solutions of the homogeneous $4^{\text {th }}$-order difference equation

$$
u(k+2)-2 u(k+1)+2 u(k-1)+u(k-2)=0, \quad k \in \mathbb{Z}
$$

with the respective initial conditions

$$
\begin{aligned}
& x(-1)=1, x(0)=0, x(1)=1, x(2)=1 \\
& y(-1)=0, y(0)=0, y(1)=0, y(2)=1 .
\end{aligned}
$$

We can solve the above problems using Mathematica ${ }^{\circledR}$. By means of the RSolve command, after arranging the obtained results, we eventually get

$$
\begin{gathered}
\operatorname{Exp} \operatorname{Cos} F(k)=\frac{\sqrt{2-\mathrm{i}}\left(\Psi_{1}^{k}-\psi_{1}^{k}\right)+\sqrt{2+\mathrm{i}}\left(\Psi_{2}^{k}-\psi_{2}^{k}\right)}{2 \sqrt{10}} \\
\operatorname{Exp} \operatorname{Sin} F(k)=\frac{\sqrt{-2+\mathrm{i}}\left(\psi_{1}^{k}-\Psi_{1}^{k}\right)+\sqrt{-2-\mathrm{i}}\left(\psi_{2}^{k}-\Psi_{2}^{k}\right)}{2 \sqrt{10}},
\end{gathered}
$$

where $k \in \mathbb{Z}$ and

$$
\Psi_{1,2}:=\left(\frac{1}{2} \pm \frac{1}{2} \mathrm{i}\right)+\sqrt{1 \pm \frac{1}{2} \mathrm{i}}, \quad \psi_{1,2}:=\left(\frac{1}{2} \pm \frac{1}{2} \mathrm{i}\right)-\sqrt{1 \pm \frac{1}{2} \mathrm{i}} .
$$

By using Mathematica ${ }^{\circledR}$, we can also determine any terms of the sequences (32), (33). The below tables pertain to the range $k \in \overline{-10,12}$ :

\begin{tabular}{c|cccccccccccccc}
$k$ & $\ldots$ & -10 & -9 & -8 & -7 & -6 & -5 & -4 & -3 & -2 & -1 & 0 \\
\hline $\operatorname{Exp} \operatorname{Cos} F(k)$ & $\ldots$ & 39 & -43 & 32 & -19 & 9 & -3 & 0 & 1 & -1 & 1 & 0 \\
$k$ & 1 & 2 & 3 & 4 & 5 & 6 & 7 & 8 & 9 & 10 & 11 & 12 & $\ldots$ \\
\hline $\operatorname{Exp} \operatorname{Cos} F(k)$ & 1 & 1 & 1 & 0 & -3 & -9 & -19 & -32 & -43 & -39 & 5 & 128 & $\ldots$ \\
$k$ & $\ldots$ & -10 & -9 & -8 & -7 & -6 & -5 & -4 & -3 & -2 & -1 & 0 \\
\hline $\operatorname{Exp} \operatorname{Sin} F(k)$ & $\ldots$ & 87 & -36 & 8 & 4 & -7 & 6 & -4 & 2 & -1 & 0 & 0 \\
$k$ & 1 & 2 & 3 & 4 & 5 & 6 & 7 & 8 & 9 & 10 & 11 & 12 & $\ldots$ \\
\hline $\operatorname{Exp} \operatorname{Sin} F(k)$ & 0 & 1 & 2 & 4 & 6 & 7 & 4 & -8 & -36 & -87 & -162 & -244 & $\ldots$
\end{tabular}


It is also worth noticing that $\operatorname{Exp} \operatorname{Cos} F(k)$ and $\operatorname{Exp} \operatorname{Sin} F(k)$ for $k \in \mathbb{N}_{0}$ are presented in the $O E I S^{\circledR 9}$ database as sequences A143056 and A272665.
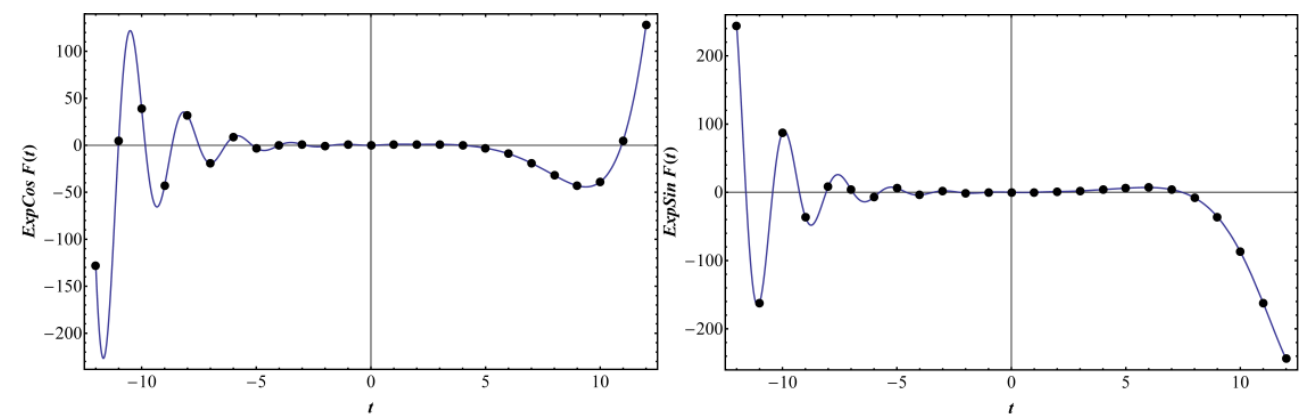

Fig. 1. Graphs of Fibonacci exponential-trigonometric functions and sequences

If we apply the Re, ComplexExpand and FullSimplify Mathematica ${ }^{\circledR}$ commands to the formulas (32), (33), then, after arranging the obtained results, we get the mentioned sequences in real forms. Namely,

$$
\begin{aligned}
\operatorname{Exp} \operatorname{Cos} F(k) & =\frac{1}{\sqrt{2 \sqrt{5}}}\left[\sqrt{\left(\Phi_{1}+\sqrt{\Phi_{1}}\right)^{k}} \cos \left(\frac{1}{2} \operatorname{arccot}(2)-k \arctan \left(\sqrt{\Phi_{1}^{-3}}\right)\right)\right. \\
& \left.-\sqrt{\left(\Phi_{1}-\sqrt{\Phi_{1}}\right)^{k}} \cos \left(\frac{1}{2} \operatorname{arccot}(2)-k\left(\pi-\arctan \left(\sqrt{\Phi_{1}^{-3}}\right)\right)\right)\right] \\
\operatorname{Exp} \operatorname{Sin} F(k) & =\frac{1}{\sqrt{2 \sqrt{5}}}\left[\sqrt{\left(\Phi_{1}-\sqrt{\Phi_{1}}\right)^{k}} \sin \left(\frac{1}{2} \operatorname{arccot}(2)-k\left(\pi-\arctan \left(\sqrt{\Phi_{1}^{-3}}\right)\right)\right)\right] \\
& \left.-\sqrt{\left(\Phi_{1}+\sqrt{\Phi_{1}}\right)^{k}} \sin \left(\frac{1}{2} \operatorname{arccot}(2)-k \arctan \left(\sqrt{\Phi_{1}^{-3}}\right)\right)\right],
\end{aligned}
$$

where $k \in \mathbb{Z}$.

A classic logarithmic spiral in a complex plane is a graph of the function

$$
z(t)=e^{(\alpha+\beta \mathrm{i}) t} .
$$

On the basis of the Euler-Fibonacci formula (31), the $(1+i)$-Fibonacci numbers can be also interpreted as complex plane points

$$
z_{k}:=(\operatorname{Exp} \operatorname{Cos} F(k), \operatorname{Exp} \operatorname{Sin} F(k)), \quad k \in \mathbb{Z},
$$

${ }^{9}$ OEIS ${ }^{\circledR}$ - The On-Line Encyclopedia of Integer Sequences ${ }^{\circledR}$, https://oeis.org/. 
lying on the $(1+i)$-Fibonacci spiral (fig. 2) with a parametric description $x(t):=\operatorname{Exp} \operatorname{Cos} F(t), \quad y(t):=\operatorname{Exp} \operatorname{Sin} F(t), \quad t \in \mathbb{R}$.
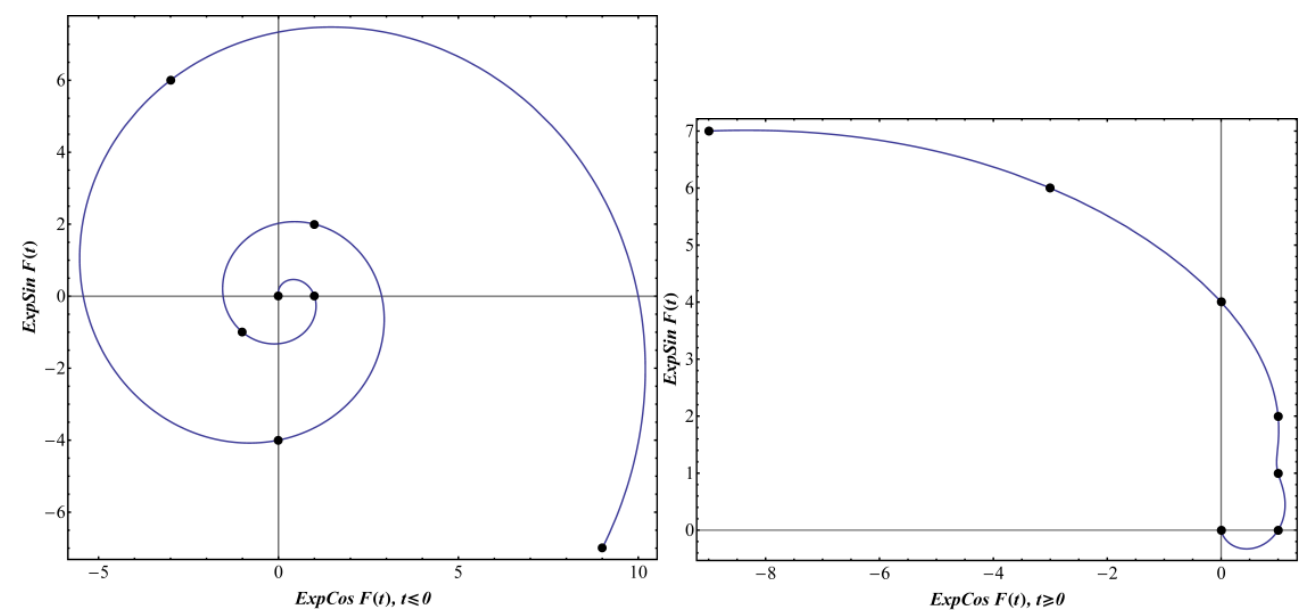

Fig. 2. Fibonacci spiral and $(1+i)$-Fibonacci numbers

The trigonometric Fibonacci sequences $x=\{\operatorname{Cos} F(k)\}$ and $y=\{\operatorname{Sin} F(k)\}$ are obtained from (28)-(30) for $\alpha=0$ and $\beta=1$, i.e. for $\lambda:=i$. Thus, $x$ and $y$ fulfil the equation

$$
u(k+2)-u(k)+u(k-2)=0, \quad k \in \mathbb{Z}
$$

and initial conditions

$$
\begin{aligned}
& x(-1)=1, x(0)=0, x(1)=1, x(2)=0 \\
& y(-1)=0, y(0)=0, y(1)=0, y(2)=1
\end{aligned},
$$

taking the forms of

$$
\begin{aligned}
& \operatorname{Cos} F(k)=\frac{1}{\sqrt{3}}\left[\cos \left(\frac{k \pi}{6}\right)-\cos \left(\frac{5 k \pi}{6}\right)\right]=\frac{2}{\sqrt{3}} \sin \left(\frac{k \pi}{2}\right) \sin \left(\frac{k \pi}{3}\right) \\
& \operatorname{Sin} F(k)=\frac{1}{\sqrt{3}}\left[\sin \left(\frac{k \pi}{6}\right)-\sin \left(\frac{5 k \pi}{6}\right)\right]=-\frac{2}{\sqrt{3}} \sin \left(\frac{k \pi}{3}\right) \cos \left(\frac{k \pi}{2}\right)
\end{aligned}
$$

It is easy to verify that

$$
\begin{gathered}
\operatorname{Cos} F(-k)=\operatorname{Cos} F(k)=-\operatorname{Cos} F(k+6) \\
\operatorname{Sin} F(-k)=-\operatorname{Sin} F(k)=\operatorname{Sin} F(k+6) \quad, \quad k \in \mathbb{Z} . \\
\operatorname{Cos} F(k+3)=-\operatorname{Sin} F(k), \operatorname{Sin} F(k+3)=\operatorname{Cos} F(k)
\end{gathered}
$$


We also have

$\operatorname{Cos} F(k+12)=\operatorname{Cos} F(k), \quad \operatorname{Sin} F(k+12)=\operatorname{Sin} F(k), \quad k \in \mathbb{Z}$.

Thus, the sequences (34), (35) are 12-periodic. The following tables present their cycles, i.e. values for $k \in \overline{0,11}$ :

\begin{tabular}{c|rrrrrrrrrrrr}
$k$ & 0 & 1 & 2 & 3 & 4 & 5 & 6 & 7 & 8 & 9 & 10 & 11 \\
\hline $\operatorname{Cos} F(k)$ & 0 & 1 & 0 & 0 & 0 & -1 & 0 & -1 & 0 & 0 & 0 & 1 \\
$k$ & 0 & 1 & 2 & 3 & 4 & 5 & 6 & 7 & 8 & 9 & 10 & 11 \\
\hline $\operatorname{Sin} F(k)$ & 0 & 0 & 1 & 0 & 1 & 0 & 0 & 0 & -1 & 0 & -1 & 0
\end{tabular}
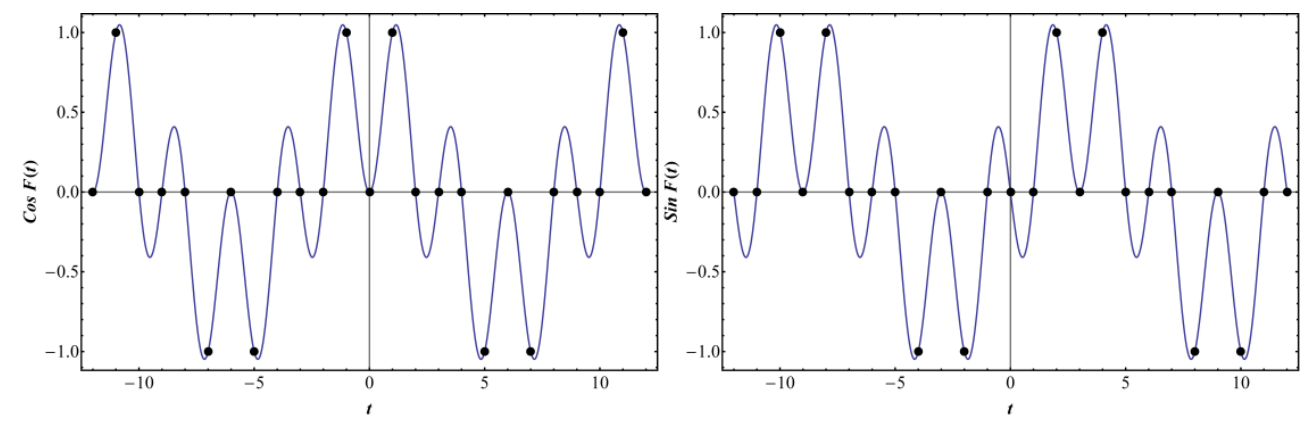

Fig. 3. Graphs of Fibonacci trigonometric functions and sequences

The sequence $\operatorname{Cos} F(k), k \in \mathbb{N}_{0}$ can be found in $O E I S^{\circledR}$ under A110161.

Fig. 4 presents a graph of the Fibonacci circle

$$
x(t):=\operatorname{Cos} F(t), \quad y(t):=\operatorname{Sin} F(t), \quad t \in[0,12) .
$$

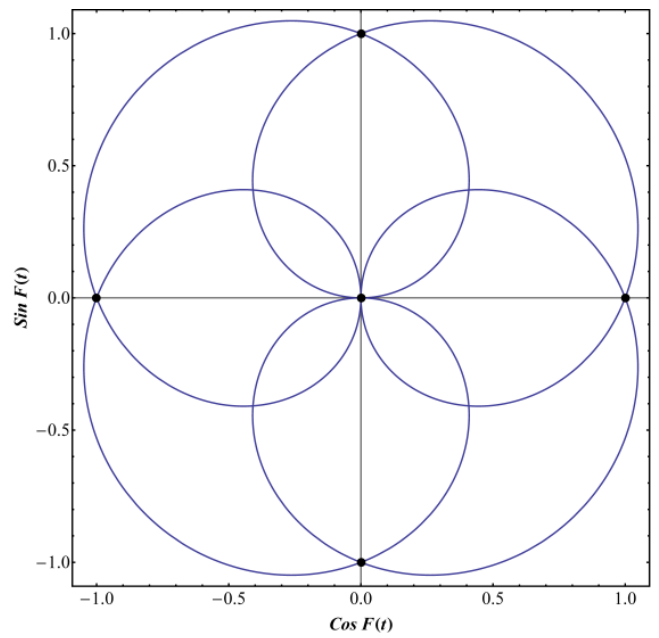

Fig. 4. Fibonacci circle 
Using (34) and (35), we obtain the Fibonacci trigonometric identity

$$
\operatorname{Cos}^{2} F(k)+\operatorname{Sin}^{2} F(k)=F(k)(\bmod 2), \quad k \in \mathbb{Z},
$$

where $F(k)(\bmod 2)=\frac{4}{3} \sin ^{2}\left(\frac{k \pi}{3}\right), k \in \mathbb{Z}$ is a 3 -periodic sequence 10

$$
\ldots, 0,1,1,0,1,1,0,1,1, \ldots \text {. }
$$

It is not difficult to verify that

$$
S\{\operatorname{Cos} F(k)\}=-\{\operatorname{Sin} F(k)\}, \quad S\{\operatorname{Sin} F(k)\}=\{\operatorname{Cos} F(k)\},
$$

where $S \equiv D_{1}$ is the central difference (21).

From (27) we have

$$
s_{0}\{\operatorname{Cos} F(k)\}=\left\{\frac{1}{2}\left(1-(-1)^{k}\right\}, \quad s_{0}\{\operatorname{Sin} F(k)\}=\{0\},\right.
$$

where $s_{0}$ is the limit condition (23) for $k_{0}=0$.

From (36) and (37) we get in turn

$$
T_{0}\{\operatorname{Cos} F(k)\}=\{\operatorname{Sin} F(k)\}, \quad T_{0}\{\operatorname{Sin} F(k)\}=-\{\operatorname{Cos} F(k)\}+\left\{\frac{1}{2}\left(1-(-1)^{k}\right\},\right.
$$

where $T_{0}$ is the integral (22) for $k_{0}=0$.

The unilateral $\mathcal{Z}$-transform for one-sided sequences

$$
x=\{\operatorname{Cos} F(k)\}_{k \in \mathbb{N}_{0}}, \quad y=\{\operatorname{Sin} F(k)\}_{k \in \mathbb{N}_{0}}
$$

is equal to

$$
X(z)=\frac{z^{3}-z}{z^{4}-z^{2}+1}, \quad Y(z)=\frac{z^{2}}{z^{4}-z^{2}+1},
$$

respectively. Therefore,

$$
G_{c}(z):=X\left(\frac{1}{z}\right)=-X(z) \text { and } G_{s}(z):=Y\left(\frac{1}{z}\right)=Y(z)
$$

are generating functions of these sequences. Hence

$$
G_{c}(z)=\frac{-z^{3}+z}{z^{4}-z^{2}+1}=\sum_{k=0}^{\infty} \operatorname{Cos} F(k) z^{k}
$$

and

$$
G_{s}(z)=\frac{z^{2}}{z^{4}-z^{2}+1}=\sum_{k=0}^{\infty} \operatorname{Sin} F(k) z^{k}
$$

10 It is a sequence $\mathrm{A} 011655$ for $k \in \mathbb{N}_{0}$ (see also [22]).

3 (214) 2018 
In both cases the denominator

$$
W(z)=z^{4}-z^{2}+1
$$

is a $12^{\text {th }}$-cyclotomic polynomial, i.e. $W(z)=P_{12}(z)$, where

$$
P_{n}(z):=\prod_{j \in \mathbb{A}_{n}}\left(z-\xi_{j}\right)
$$

and $\xi_{j}:=\cos \frac{2 j \pi}{n}+\mathrm{i} \sin \frac{2 j \pi}{n}, \mathbb{A}_{n}:=\{j \in \overline{1, n}:(j, n)=1\}$.

\section{$(b, \lambda, 2 n)$-BONACCI SEQUENCES}

A two-sided sequence $z=\{z(k)\} \in C(\mathbb{Z}, \mathbb{C})$, satisfying the equation

$$
z(k+n)-b z(k-n)=\lambda z(k), \quad b, \lambda \in \mathbb{C} \backslash\{0\}
$$

as well as the initial conditions

$$
z\left(k_{0}\right)=c_{k_{0}}, z\left(k_{0}+1\right)=c_{k_{0}+1}, \ldots, z\left(k_{0}+2 n-1\right)=c_{k_{0}+2 n-1}
$$

will be called a $(b, \lambda, 2 n)$-Bonacci sequence.

In the operational calculus model (20) it is an exponential sequence fulfilling the equation

$$
S_{b} z=\lambda z
$$

and the limit condition

$$
s_{b, k_{0}} z=c=\left\{\frac{\sqrt[2 n]{b^{k}}}{2 n} \sum_{j=0}^{2 n-1} \sum_{i=k_{0}}^{k_{0}+2 n-1} \varepsilon_{j}^{k-i} \frac{c_{i}}{\sqrt[2 n]{b^{i}}}\right\}
$$

In [11], Kalman and Mena considered one-sided $(b, \lambda, 2)$-Bonacci sequences, when $k_{0}=0$ and $b, \lambda \in \mathbb{R} \backslash\{0\}$.

A $(1,1,2 n)$-Bonacci sequence $\mathcal{F}=\{\mathcal{F}(k)\}$, of which $2 n$ consecutive terms are Fibonacci numbers

$$
\mathcal{F}(k):=F(k)=\frac{\Phi_{1}^{k}-\varphi_{1}^{k}}{\Phi_{1}-\varphi_{1}}=\frac{1}{\sqrt{5}}\left[\left(\frac{1+\sqrt{5}}{2}\right)^{k}-\left(\frac{1-\sqrt{5}}{2}\right)^{k}\right], \quad k \in \overline{0,2 n-1}
$$

will be called a ( $2 n)$-Fibonacci sequence.

For example, a (4)-Fibonacci sequence is a solution of the equation

$$
\mathcal{F}(k+2)-\mathcal{F}(k-2)=\mathcal{F}(k)
$$

with conditions

$$
\mathcal{F}(0)=F(0)=0, \mathcal{F}(1)=F(1)=1, \mathcal{F}(2)=F(2)=1, \mathcal{F}(3)=F(3)=2
$$

60

Scientific Journal of PNA — Zeszyty Naukowe AMW 
Using Mathematica ${ }^{\circledR}$, we obtain a solution of the problem (38), (39):

$$
\begin{gathered}
\mathcal{F}(k)=\frac{1}{\sqrt{5}}\left[\left(\sqrt{\Phi_{1}^{k}} \cos \left(\frac{k \pi}{2}\right)-\sqrt{\Phi_{1}^{-k}}\right) \cos \left(\frac{k \pi}{2}\right)\right. \\
\left.+\left(\sqrt{\Phi_{1}^{k+3}} \sin \left(\frac{k \pi}{2}\right)-\sqrt{\Phi_{1}^{-(k+3)}}\right) \sin \left(\frac{k \pi}{2}\right)\right] .
\end{gathered}
$$

Hence, we have

$$
\mathcal{F}(2 k)=F(k), \mathcal{F}(2 k-1)=F(k+1), \quad k \in \mathbb{Z} .
$$

The sequence $\mathcal{F}(k), k \in \mathbb{N}_{0}$ can be found in $O E I S^{\circledR}$ under A053602:

\begin{tabular}{c|cccccccccccccccccc}
$k$ & 0 & 1 & 2 & 3 & 4 & 5 & 6 & 7 & 8 & 9 & 10 & 11 & 12 & 13 & 14 & 15 & 16 & $\ldots$ \\
\hline $\mathcal{F}(k)$ & 0 & 1 & 1 & 2 & 1 & 3 & 2 & 5 & 3 & 8 & 5 & 13 & 8 & 21 & 13 & 34 & 21 & $\ldots$
\end{tabular}

\section{REFERENCES}

[1] Apostol T. M., Calculus, Vol. 1, One-Variable Calculus, with an Introduction to Linear Algebra, John Wiley \& Sons, New York — London 1967.

[2] Bittner R., On certain axiomatics for the operational calculus, 'Bull. Acad. Polon. Sci.', Cl. III, 1959, 7(1), pp. 1-9.

[3] Bittner R., Operational calculus in linear spaces, 'Studia Math.', 1961, 20, pp. 1-18.

[4] Bittner R., Algebraic and analytic properties of solutions of abstract differential equations, 'Rozprawy Matematyczne' ['Dissertationes Math.'], 42, PWN, Warszawa 1964.

[5] Bittner R., Rachunek operatorów w przestrzeniach liniowych, PWN, Warszawa 1974 [Operational Calculus in Linear Spaces — available in Polish].

[6] Bittner R., Mieloszyk E., About eigenvalues of differential equations in the operational calculus, 'Zeszyty Naukowe Politechniki Gdańskiej, Matematyka XI', 1978, 285, pp. 87-99.

[7] Elaydi S., An Introduction to Difference Equations, Springer Sci. \& Business Media, New York 2005.

[8] Falcón S., Plaza A., On the Fibonacci k-numbers, 'Chaos, Solitons and Fractals', 2007, 32(5), pp. 1615-1624.

[9] Gazalé M. J., Gnomon: From Pharaohs to Fractals, Princeton Univ. Press, New Jersey 1999.

[10] Jordan Ch., Calculus of Finite Differences, Chelsea Publ. Comp., New York 1950.

[11] Kalman D., Mena R., The Fibonacci numbers - exposed, 'Math. Magazine', 2003, 76(3), pp. 167-181.

[12] Levy H., Lessman F., Finite Difference Equations, Pitman \& Sons, London 1959.

[13] Mathews J. H., Fink K. D., Numerical Methods Using MATLAB, Prentice Hall, New Jersey 1999.

[14] Mercer P. R., More Calculus for a Single Variable, Springer Sci. \& Business Media, New York 2014.

[15] Mikusiński J., Operational Calculus, Pergamon Press, London 1959.

3 (214) 2018 
[16] Spinadel V. W., The Family of Metallic Means, 'VisMath — Visual Mathematics', Electronic Journal, 1999, Vol. 1(3) [online], http://www.mi.sanu.ac.rs/vismath/spinadel/index.html [access 14.06.2018].

[17] Spinadel V. W., New Smarandache Sequences: The Family of Metallic Means [online], http://vixra.org/abs/1403.0507 [access 14.06.2018].

[18] Stakhov A., The Mathematics of Harmony: From Euclid to Contemporary Mathematics and Computer Science, Series of Knots and Everything: Vol. 22, World Scientific, Singapore 2009.

[19] Stakhov A., Rozin B., On a new class of hyperbolic functions, 'Chaos, Solitons and Fractals', 2005, 23(2), pp. 379-389.

[20] Washburn L., The Lanczos derivative, Senior Project Archive 2006, Dept. of Maths., Whitman College, USA, [online], https://www.whitman.edu/Documents/Academics/Mathematics/washbuea.pdf [access 14.06.2018].

[21] Wysocki H., Spira Mirabilis in the selected models of the Bittner operational calculus, 'Zeszyty Naukowe Akademii Marynarki Wojennej' ['Scientific Journal of PNA'], 2015, 4(203), pp. 65-96.

[22] Wysocki H., An operational calculus model for the $n^{\text {th }}$-order forward difference, 'Zeszyty Naukowe Akademii Marynarki Wojennej' ['Scientific Journal of PNA'], 2017, 3(210), pp. 107-117.

\section{MODEL RACHUNKU OPERATORÓW DLA RÓŻNICY CENTRALNEJ \\ ORAZ WYKŁADNICZO - TRYGONOMETRYCZNE I HIPERBOLICZNE CIĄG I FIBONACCIEGO}

\section{STRESZCZENIE}

W artykule skonstruowano model nieklasycznego rachunku operatorów Bittnera, w którym pochodna rozumiana jest jako różnica centralna $D_{n}\{x(k)\}:=\{x(k+n)-x(k-n)\}$. Dokonano uogólnienia opracowanego modelu, rozważając operację $D_{n, b}\{x(k)\}:=\{x(k+n)-b x(k-n)\}$, gdzie $b \in \mathbb{C} \backslash\{0\}$. W modelu z różnicą $D_{1}$ wprowadzono wykładniczo-trygonometryczne i hiperboliczne ciągi Fibonacciego.

Słowa kluczowe:

rachunek operatorów, pochodna, pierwotne, warunki graniczne, różnica centralna, ciągi Fibonacciego.

Article history

Received: 14.06 .2018

Reviewed: 17.09 .2018

Revised: $\quad 19.09 .2018$

Accepted: 20.09.2018 